P1: FQF/FGC P2: FQF

0521833337c19.xml CUUK009-Li et al. September 8, 2005 11:34

\title{
19 Phonological mediation in visual word recognition in English and Chinese
}

In-mao Liu, Jei-tun Wu, Iue-ruey Sue, and Sau-chin Chen

This study examines the role of phonological information in the visual recognition of written words. The term visual word recognition is used here to refer to the process of word-form identification as opposed to meaning retrieval (Monsell, Doyle \& Haggard, 1989). Meaning retrieval implies word-form identification, however, not vice versa.

Phonological information could be generated from the visually presented word in two ways. First, the word is recognized as a particular orthographic pattern and then its pronunciation retrieved from memory (addressed phonology). Second, phonological information concerning the word can be generated via spelling-sound correspondences during the recognition process before identification is complete, which represents an instance of assembled phonology (Patterson \& Coltheart, 1987). The assembled phonology route necessarily assumes that component letters or graphemes be identified to an extent that allows spelling-sound correspondence rules to be applicable. Evidence for phonological mediation naturally consists of demonstrating that the word's graphemic units activate phonemic units earlier than the word is recognized. Several methods have been used to claim evidence for phonological mediation.

\section{Paradigms used for studying phonological mediation}

Two paradigms have generally been used to provide evidence for, or against, phonological mediation in English and Chinese. They are the priming paradigm and semantic categorization paradigm.

\section{Priming paradigm}

There are English and Chinese studies using the priming paradigm. Although the priming paradigm used in Chinese studies differs in some details from the priming paradigm used in English studies, both studies aimed at showing that phonological activation is very early. The logic of very early phonological

* This study was supported by National Science Council Grant 91-2413-H-194-004-. 
P1: FQF/FGC P2: FQF

0521833337c19.xml CUUK009-Li et al. September 8, 2005 11:34

activation is based on the observation that the prime-target SOA (stimulus onset asynchrony) is very short and yet capable of producing priming effects.

English studies. Using a primed perceptual task, Perfetti and Bell (1991) found that correct identification of targets primed by pseudohomophone primes (e.g. mayd-MADE) was significantly higher than that obtained with orthographic controls (e.g. mard-M ADE). This effect is accounted for in terms of a rapid activation of phonemic units by the corresponding graphemic units, these phonemic units in turn increasing the activation level of any word units that contain them. Ferrand and Grainger (1992) have observed the same effects in a primed lexical decision task. Lukatela and his associates (Lukatela, Carello, \& Turvey, 1990; Lukatela \& Turvey, 1990a, b) have also obtained phonological priming effects in Serbo-Croatian in similar conditions with both the perceptual identification and lexical decision tasks.

The inference of very early phonological activation from the priming effect observed at very short SOAs assumes that the prime's graphemic units rapidly activate phonemic units, because the prime duration is as short as $45 \mathrm{~ms}$. Available evidence (Berent \& Perfetti, 1995) showed that the word duration is inversely related to the naming latency. In the standard naming condition in Berent and Perfetti's Experiment 5a-b, they observed the mean naming time of approximately $560 \mathrm{~ms}$ for the target's exposure duration of $900 \mathrm{~ms}$. In the brief naming condition (target's exposure duration of $60 \mathrm{~ms}$ ) they observed the mean naming time of about $610 \mathrm{~ms}$, even though negative feedback was given to the participant' slow reaction times exceeding $1000 \mathrm{~ms}$ (5.6 percent of the total correct responses in the brief naming condition, but only 0.9 percent in the standard naming condition). Since it is assumed that the time of phonological activation is directly related to the naming latency, it follows that the word duration is inversely related to the time of phonological activation.

Chinese studies Noncharacters being not pronounceable, homophones, and semantically related characters (or two-character words) are generally used as the priming stimuli in Chinese studies. The aim of these priming studies was to ascertain which prime type, the homophone prime or semantic prime, produces a larger priming effect at a shorter prime duration. Perfetti and Zhang (1991) observed a homophone priming effect as large as a semantic priming effect at a $50-\mathrm{ms}$ prime duration. In this experiment (Experiment 3), the target was exposed for $35 \mathrm{~ms}$.

In Perfetti and Tan (1998), the target remained on the screen until participants made a response. They observed a homophone priming effect at the 57-ms SOA, but were unable to observe a semantic priming effect until the 85-ms SOA. Perfetti and Tan concluded, "graphic information was activated first, within $43 \mathrm{~ms}$, followed by phonological information within $57 \mathrm{~ms}$ and by semantic information within $85 \mathrm{~ms}$ " (1998: 111). It should be noted that both Chen and Shu (2001) and Wu and Chen (2000) obtained a contradictory finding. 
P1: FQF/FGC P2: FQF

0521833337c19.xml CUUK009-Li et al. September 8, 2005 11:34

Recently Zhou and Marslen-Wilson (2000) measured homophone "yes" response latencies as a function of prime SOAs in a homophone judgment task (Experiment 4):

$\begin{array}{ll}\text { SOA } & \text { Homophone "yes" response latency } \\ 57 \mathrm{~ms} & 714 \mathrm{~ms} \\ 86 \mathrm{~ms} & 687 \mathrm{~ms} \\ 200 \mathrm{~ms} & 563 \mathrm{~ms}\end{array}$

Their targets lasted for $400 \mathrm{~ms}$. It can be seen that the SOA is inversely related to the response latency. However, with these data they concluded, "indeed, the fact that most participants could make positive responses to homophones at the SOA of $57 \mathrm{~ms}$ indicates that phonological activation can occur very early" (2000: 1258). Again, their conclusion is based on an assumption that, whenever there is a phonological priming effect, phonological activation must have taken place within the SOA. Their conclusion apparently contradicts their own finding of an inverse relationship between the SOA and response latency.

\section{Semantic categorization paradigm}

Recently, Taft, and van Graan (1998) interpreted the issue of phonological mediation to mean whether the phonological information mediates between the orthographic information and semantic information. This is not the usual way in which the issue of phonological mediation is understood, as we can see from the very beginning sentences of van Orden's (1987) influential article:

The possibility that the sound (or pronunciation) of words influences the identification of printed words has been repeatedly both affirmed and denied throughout the history of the study of the psychology of reading. This possibility, usually termed "phonological mediation," assumes that there are phonological sources of activation that precede word identification.

In van Orden's (1987) Experiment 1, the category name and fixation point remained visible for $1,500 \mathrm{~ms}$ and then were replaced by a target word. The target word was visible for $500 \mathrm{~ms}$ before a pattern mask replaced it. Upon presentation of the target word, the participants" task was to respond orally "yes" or "no", indicating "yes, it is a category exemplar" or "no, it is not," and then to name the target word. He found that participants produced larger false positive error rates in a semantic categorization task when they responded to stimulus foils that were homophonic to category exemplars (e.g. Rows for the category A FLOWER) than when they responded to spelling control foils. He found a larger homophony effect under brief-exposure pattern-masking conditions in Experiment 2. 
P1: FQF/FGC P2: FQF

0521833337c19.xml CUUK009-Li et al. September 8, 2005 11:34

Phonological mediation in visual word recognition

A higher false alarm rate to homophones of category members than to orthographically similar controls is now well documented (e.g. Banks, Oka, \& Shugarman, 1981; Coltheart, Patterson, \& Leahy, 1994; Peter \& Turvey, 1994; Van Orden, Johnston \& Hale, 1988). The semantic categorization task involving homophones must be a difficult task, as Van Orden (1987) found the mean categorization times to be from $900 \mathrm{~ms}$ to $1100 \mathrm{~ms}$ in his Experiment 3 .

Although spelling controls are usually used in the semantic categorization task, both homophone (ROW ) and its control (R O B S) share three letters with the category member (ROSE) in English. In Chinese, on the other hand, two characters share no component and yet can be pronounced the same. In this case, a higher false alarm rate to homophones of category members than to orthographically similar controls was not observed (Chen, Flores D'Arcais \& Cheung, 1995; Leck, Weekes, \& Chen, 1995; Wydell, Patterson \& Humphreys, 1993).

\section{Other paradigms}

The masking paradigm is similar to the priming paradigm. In the masking paradigm, a target word is presented for a brief moment and then replaced by a word or nonword mask and a visual pattern. The task is target identification. As in the priming and semantic categorization paradigms, these paradigms all involve more than one word and all stimuli tend to be presented for a brief period. In this visually impoverished situation, participants naturally tend to rely more on phonological than on orthographic information. However, no study has clearly demonstrated that a word's graphemic units activate phonemic units earlier than the word is recognized.

There is another issue of whether phonology mediates between orthographic information (i.e. word identification) and semantic information. Investigators have used various tasks for studying this problem: semantic discrimination (e.g. Luo, 1996), semantic priming (e.g. Lesch \& Pollatsek, 1993; Lukatela, Lukatela \& Turvey, 1993), sentence acceptability (e.g. Baron, 1973), and semantic and phonological judgments (e.g. Perfetti \& Zhang, 1995). The last paradigm involving semantic and phonological judgments includes two tasks. In the semantic judgment task, participants had to decide whether the paired characters were synonyms, whereas in the phonological judgment task, they were to judge whether the paired characters were homophones. By varying the stimulus onset asynchronies (SOAs) between the first and second characters, they found that phonological interference with synonym judgments appeared at the SOA of $90 \mathrm{~ms}$ or longer, but that semantic interference effect with homophone judgments did not appear until the SOA was $140 \mathrm{~ms}$. In interpreting their findings, Perfetti and Zhang wrote as follows, "Is Chinese read by a process that bypasses phonology, or is phonology a part of word identification as it is an 
P1: FQF/FGC P2: FQF

0521833337c19.xml CUUK009-Li et al. September 8, 2005 11:34

alphabetic writing systems? Two experiments provide evidence that phonological information is activated as part of Chinese character identification."

The observation of phonological interference in semantic judgments is unable to settle the issue of phonological mediation, because activation of the semantic code takes place after a word is identified (Monsell, Doyle \& Haggard, 1989). Thus, the finding that phonological activation precedes semantic activation does not necessarily mean that the former also precedes word identification.

\section{A matching paradigm for studying phonological mediation}

Evidence for phonological mediation consists of demonstrating that the word's graphemic units activate phonemic units earlier than the word is recognized. For the word's graphemic units to be capable of activating phonemic units, the graphemic units must be categorized. The existence of grapheme-phoneme correspondence rules defines the grapheme categorization. Thus, phonological mediation presupposes that the word's graphemic units are categorized before the word itself is categorized. In other words, the phonological mediation hypothesis presupposes that the word's component letters or graphemes are identified before the word itself is identified.

When using Chinese characters, the phonetic-sound correspondences are comparable to the grapheme-phoneme correspondences in alphabetic languages. A Chinese version of the phonological mediation hypothesis becomes: participants identify embedded phonetics faster than whole characters, because they compute the phonetic-sound correspondences in pronouncing characters faster than they identify words.

\section{Method}

The present experiment used a matching paradigm, which is a variation of Reicher's procedure (Reicher, 1969), for finding the time course of identifying characters and embedded phonetics. In each trial of this procedure, a target item was presented with two alternative items. The task was to choose one of the two alternatives that better matched the target. There were three conditions. In the character condition, two characters (e.g. 防, 阱) were alternatives, and the task was to choose the one (防) that was identical to the target character (e.g. 防). In the phonetic-character condition, two phonetics printed in character fonts (方, 井) were alternatives, and the task was to choose the one (方) that matched the phonetic embedded in the target character (防). It should be noted that a phonetic embedded in a character usually occupies the right half of a character space. Such a phonetic is, therefore, printed in a horizontally compressed form. In the phonetic-compressed condition, two phonetics printed in the way they are embedded in characters were alternatives, and the task was 
P1: FQF/FGC P2: FQF

0521833337c19.xml CUUK009-Li et al. September 8, $2005 \quad$ 11:34

Phonological mediation in visual word recognition

to choose the one that was identical to the phonetic of the target. It sufficed for participants in this condition to respond entirely on the basis of a physical match.

The 80 stimulus characters used as the targets had a $2 \times 2 \times 2$ factorial structure with phonetic compatibility, character frequency, and phonetic frequency being the three orthogonal factors. There was also a set of 80 characters used as the alternatives. The participants were 24 college students.

\section{Results and discussion}

We found that the mean phonetic identification time in the phonetic-character condition $(655 \mathrm{~ms})$ was $73 \mathrm{~ms}$ significantly slower than the mean character identification time in the character condition (592 ms) and $77 \mathrm{~ms}$ significantly slower than the mean phonetic identification time in the phonetic-compressed condition $(588 \mathrm{~ms})$. We also found that the character frequency effect tended to be observed in all the three conditions. In other words, participants had generally identified characters in making responses in all the three conditions. On the other hand, the phonetic frequency effect was observed in the phonetic-character condition, but not observed in the phonetic-compressed condition. Thus, participants had identified embedded phonetics as characters in the phonetic-character condition, but had not identified embedded phonetics as characters in the phonetic-compressed condition. With respect to the error rates, it is particularly worthy to note that more errors were observed in identifying low-frequency characters than in identifying high-frequency characters in the character condition, but that this tendency was reversed in the other two conditions. Furthermore, there was no effect of phonetic frequency on error rates in the three conditions.

These results showed that participants had identified whole characters by 592 ms. Although participants had processed embedded phonetics, so that they had successfully matched the embedded phonetic with the correct alternative at this time or earlier (588 ms), they had not yet identified embedded phonetics until $655 \mathrm{~ms}$. The finding that participants cannot identify embedded phonetics faster than characters provides evidence that it is impossible to compute phoneticsound correspondences before identifying characters. In other words, there is no phonological mediation in Chinese.

\section{General discussion}

A problem with priming studies is the claim that phonology is activated during short SOAs. This claim is the reason for the conclusion of very early phonological activation. It is easy to see that this claim is wrong by presenting only the pseudohomophone primes for these short SOAs. No phonological activation 
P1: FQF/FGC P2: FQF

0521833337c19.xml CUUK009-Li et al. September 8, 2005 11:34

should be observed in this case. It is fair to conclude, however, that the priming paradigm has contributed to demonstrate that phonological information can be extracted from pseudohomophone primes and activated at later times with the following presentation of the targets, over and above the information provided by the orthographic controls.

The evidence for phonological mediation provided by using the semantic categorization paradigm in English is based on the observation of a higher false alarm rate to homophones of category members than to orthographically similar controls. As noted, phonology and spelling necessarily covary to some extent in alphabetic languages. In a difficult task involving homophones and their orthographic controls, homophones provide one additional source of correct information. It is understandable that there is a tendency to rely on phonological information in a visually impoverished situation prescribed by the semantic categorization paradigm. This may account for a higher false alarm rate to homophones of category members than to orthographically similar controls. On the other hand, a general finding that the false alarm rate to homophones of category members is far less than 50 percent shows also that participants tend to rely more on visual information than on phonological information in recognizing words. This means that phonological mediation is not universal even in alphabetic languages.

Finally, we report an experiment using a matching paradigm to test a Chinese version of the phonological mediation hypothesis that participants identify embedded phonetics faster than whole characters. The results showed that, contrary to a prediction of phonological mediation that participants compute phonetic-sound correspondences in pronouncing characters faster than they identify words, they identify characters faster than embedded phonetics.

It is clear that evidence for phonological mediation in English is observed in the semantic categorization paradigm that involves a visually impoverished situation. This evidence for phonological mediation in English is, however, not general and is restricted to the minority of cases. For Chinese, there is clear evidence using the matching paradigm that phonology is bypassed in the recognition of Chinese words. The lack of phonological activation in the recognition of words does not mean that phonology is not activated in normal reading. On the contrary, phonological activation is almost automatic following visual word recognition. 